\title{
EQUILIBRIUM OF ISOLATED MAGNETOSTATIC FLUX STRUCTURE
}

\author{
X. ZHANG and W.R. HU \\ Institute of Mechanics, Chinese Academy of Sciences, Beijing, China
}

(Received: 17 November, 1992)

\begin{abstract}
Two-dimensional magnetostatic models of flux structure confined in stratified atmosphere are discussed in the present paper. The magnetic field in the flux structure is assumed to be force-free at the first step. Numerical solutions for this nonlinear free boundary problem are obtained by finite element method. Results show clearly the relation between the inside fields and outside pressure, especially the influence of atmospheric pressure distribution on the flux structure.
\end{abstract}

\section{Introduction}

Magnetostatic equilibriums have been extensively studied in astrophysical theory for magnetic field models of continuous distribution. However, observations of solar magnetic fields suggest that, $90 \%$ magnetic flux in photosphere exists in the form of isolated flux tubes (cf. Spruit et al., 1981). It seems that the isolated fine structure of magnetic field is a challenge problem of both observation and theory research.

The simplest model of isolated flux tube is one-dimensional model, in which the flux tube is confined in a uniform atmosphere (see, for example, Parker, 1979). Two- dimensional models have been studied based on the perturbation expansion (cf. Parker, 1979; Hu et al., 1983, 1987, 1989) or on the approximation of similary solution (Deinzer, 1965; Osherovich, 1974). From view point of physics, the flux structure of solar magnetic field can be determined if solar flux is given at the photospheric boundary and the pressure distribution is given in the solar atmosphere. From view point of mathematics, this is a nonlinear problem with determined free surface, the strong nonlinear property comes from the condition of total pressure conservation at free surface, although the magnetostatic problem in the flux tube may be linear, such as the linear force free field. This is the essential difference between the continuous model and the isolated model of magnetic field.

In the present paper, we discuss the two ribbon structure of solar magnetic field described by two-dimensional returning flux configuration, that is, an arch of magnetic flux emerges from and then returns into the convection region. The case of near uniform atmospheric pressure was analyzed mathematically by weak nonlinear method, that is, the deviation of flux structure is small from a basic state of half circle arch (see Hu and $\mathrm{Li}, 1987$ ). However, the pressure of solar atmosphere decreases rapidly with the distance from photosphere, and strong nonlinear problem should be discussed (Zhang and Hu, 1992a, 1992b). 


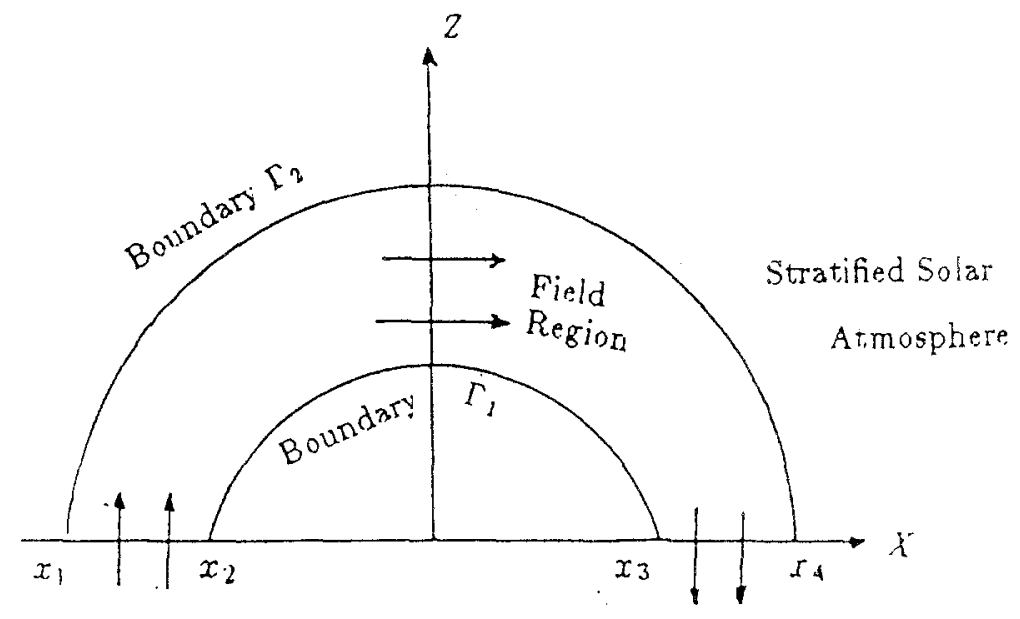

Fig. 1. Model of Magnetic Arch.

\section{Models}

We discuss a magnetic arch in Cartesian coordinates $(x, y, z)$ as shown in Fig. 1, where the $z$-axis is adopted outward, and two dimensional model requires $\partial / \partial y=$ 0 . The magnetic field is relatively strong in the arch, and may be described by the linear force-free field for simplification. The magnetic pressure outside the arch is negligible in comparison with the atmosphereic pressure. The magnetic flux confined by the solar atmosphere, and the total pressures at both side of each free boundaries $\Gamma_{1}$ and $\Gamma_{2}$ should be conservation.

The equations inside and outside the arch are given respectively by the static equilibrium conditions. We introduce the magnetic surface funtion $\psi$, the equation of linear force-free field inside arch may be written as

$$
L(\psi)=\frac{\partial^{2} \psi}{\partial x^{2}}+\frac{\partial^{2} \psi}{\partial z^{2}}+\alpha^{2} \psi=0
$$

where $\alpha$ is a constant for linear force-free field. The magnetic pressure is much smaller than the atmospheric pressure in the region outside the arch, the gradient of atmospheric pressure balances with the solar gravity $\mathrm{g}$ and it gives

$$
p_{e}(z)=p_{e}\left(z_{0}\right) \exp \left(-\int_{z_{0}}^{z}\left(g / R T_{e}\right) \mathrm{d} z\right),
$$


where $p_{e}$ and $T_{e}$ are, respectively, the atmospheric pressure and temperature in the region outside the arch and $R$ is gaseous constant. The atmospheric pressure can be given by either the experiential model based of solar observation or theoretical model based on the typical assumption. The total pressure conservation at free boundaries $\Gamma_{i}$ requires that

$$
|\nabla \psi|^{2}+\alpha^{2} \psi^{2}=P_{e}(z), \quad\left(\mathbf{r}(x, y, z) \in \Gamma_{i}, \quad i=1,2\right)
$$

Moreover, the boundary condition at photosphere $z=z_{0}$ may be described as follows:

$$
\psi\left(x, z_{0}\right)=\psi_{0}(x)
$$

The problem of isolated tlux arch is to solve Eqs. (2.1) and (2.3) under boundary conditions (2.3) and (2.4), where the configuration of boundaries $\Gamma_{1}$ and $\Gamma_{2}$, will be determined together with the solution. It should be pointed out that the constant $\alpha$ in isolated models can not be given arbitrary as in the case of continuous models. The conditions of total pressure conservation (2.3) determines the magnetic field and then the $\alpha$ values at the cross lines of photosphere $z=z_{0}$ and $\Gamma_{i}$. In this case, the value of $\alpha$ is determined for force-free field. In the present paper, the isothermal model $T_{e}=$ constant is studied and hence there is a constant atmospheric scale height $H=R T_{e} / g$.

\section{Features of Isolated Flux Arch}

The problem is solved by the finite element method, which is suitable especially for free surface problems. The equilibrium configuration of isolated magnetic flux arch in the solar atmosphere is affected by many factors, such as the external pressure, the force-free factor $\alpha$, photospheric magnetic field (as the boundary condition at $z=z_{0}$ ), the position of the foot points, etc. In order to know the infuence of these parameters more detail, we perform the following numerical experiment for axi-symmetric models, it means that the flux arch emerges from and return into the convective region in region $x_{1} \leq x \leq x_{2}$ and $x_{3} \leq x \leq x_{4}$, respectively, with $x_{3}=-x_{2}, x_{4}=-x_{1}$ in photosphere $z=z_{0}$. We let the scale non-dimensional and adopt $x_{4}=0.9$. 

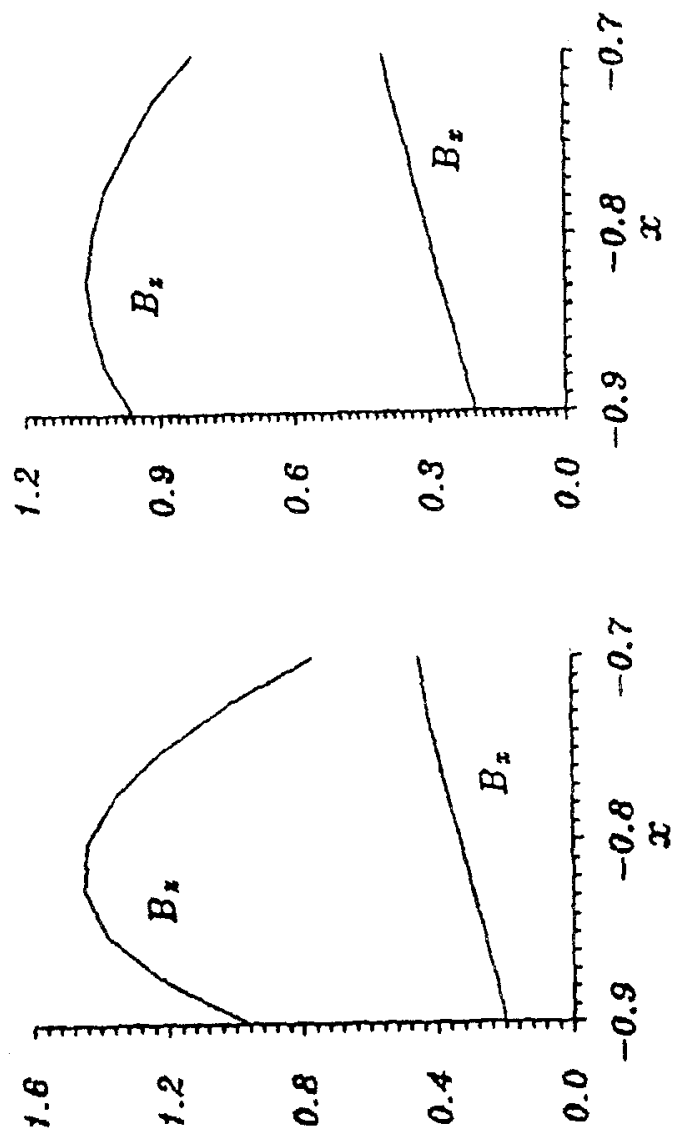

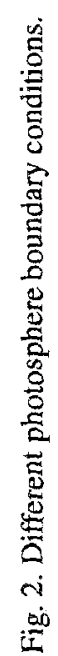

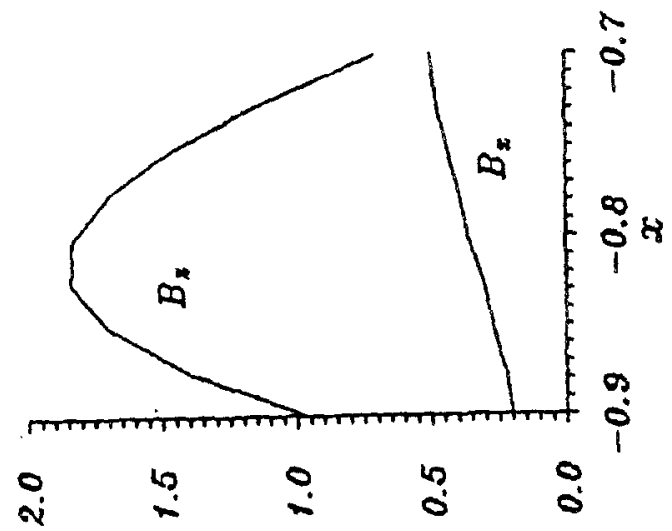



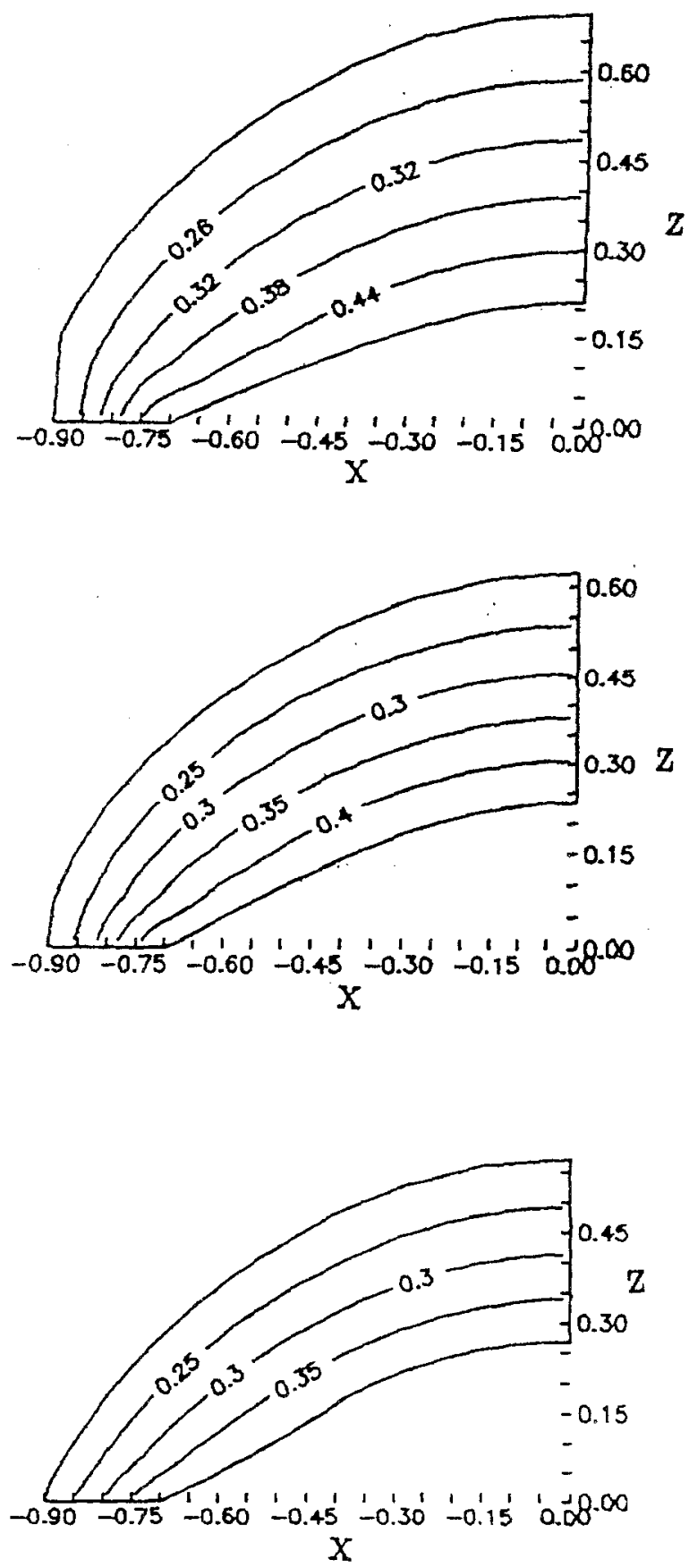

Fig. 3. Magnetic arch configurations $a, b, c$ corresponding to boundary conditions $a, b$ and $c$ in Fig. 2 for fixed $H=0.65$ and $\alpha=1$. 

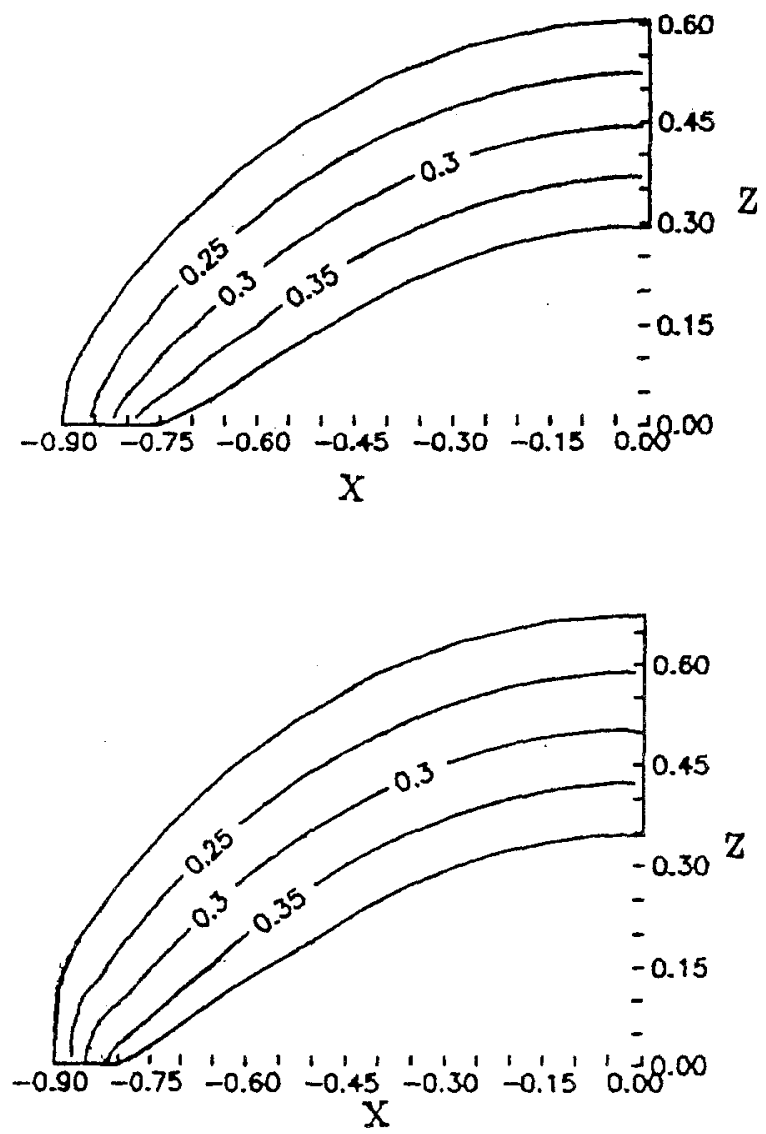

Fig. 4. Magnetic arch configurations with $x_{2}=-0.75$ (a) and -0.8 (b) respectively $(H=0.65$, $\alpha=1$ ).

\subsection{Influence OF PHOtosPheric MAGNetic Field DistRibution}

In the present paper, the magnetic field at photosphere $z=z_{0}$ has both longitudinal and transverse components $B_{z}$ and $B_{x}$, where the longitudinal component at photosphere and $x=x_{1}$ is adopted to be 0.96 and $B_{x}$ is 0.2 for non-dimension of the field. The relevant influx field are shown in Fig. 2, where the inclination angle relative to the vertical direction $\theta_{2}$ at $x_{2}$ are $35.27^{\circ}, 30.26^{\circ}$ and $25.88^{\circ}$ respectively and $\theta_{1}$ at $x_{1}$ is fixed to be $11.78^{\circ}$. Here $x_{1}, x_{2}$ and $\alpha$ are unchanged and $\tan \theta=B_{x} / B_{z}$. The results of Fig. 3 for fixed $H=0.65$ and $\alpha=1$ show that, the boundary condition of photospheric magnetic field has great influence on the configuration of the magnetic arch. As the angle $\theta_{2}$ decreases, the arch will elevate because of the reduced flux profile. 

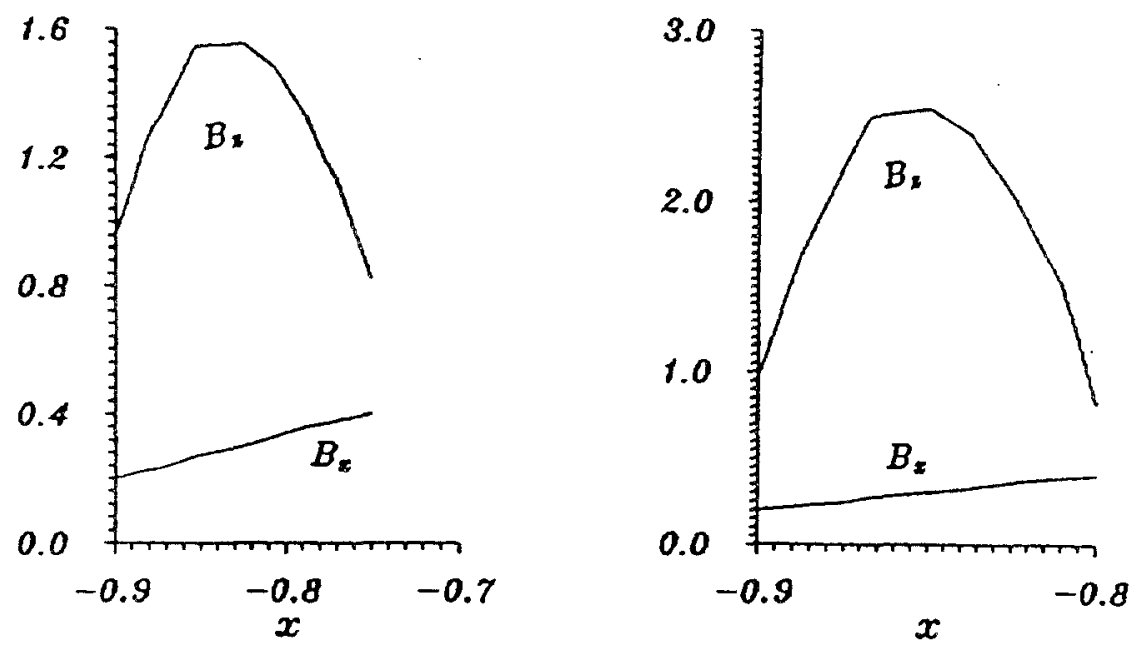

Fig. 5. Boundary conditions relating to Fig. 4.

\subsection{INFLUENCE OF FOOTPOINT POSITION}

Now we change the position of $x_{2}$ to -0.75 and -0.8 in Fig. 4 . Here, we fix also $H=0.65$ and $\alpha^{2}=1$. The related entrance conditions are shown in Fig. 5.

Comparing with Fig. $3 \mathrm{c}$, it seems that as the distance between two footpoints become closer, the arch will rise and expand because of the less of flux. In reality, when the entrance width of the flux tube is enlarged, the solar magnetic flux at the photosphere and thus the internal magnetic pressure will be increased. The external magnetic pressure have to strengthen to balance atmospheric pressure at the free boundary and thus result in the contraction of the arch.

\subsection{INFLUENCE OF ATMOSPHERIC SCALE HEIGHT $H$}

Figs. $6 \mathrm{a}, 3 \mathrm{c}, 6 \mathrm{~b}$ show the infuence of scale height $H=0.55,0.65$ and 0.8 respectively for fixed footpoint position $x_{1}=-0.9, x_{2}=-0.7$ and the force-free factor $\alpha^{2}=1$. The emerging field are the same as Fig. 2c. The results show clearly the influence of atmospheric pressure on the shape of the arch. The arch will extend more outward and fatter for the smaller scale height. In fact, scale height $H$ relates to the atmospheric pressure distribution. The outside pressure will decrease rapidly, and the pressure to confine the arch is relatively small for a lower $h$. If we define a relative width $D$ as:

$$
D=\frac{\mathrm{d} z}{\mathrm{~d} x}=\frac{\left.\left(z_{\max }-z_{\min }\right)\right|_{z=0}}{\left.\left(x_{1}-x_{2}\right)\right|_{z=0}}
$$

Figs. $6 \mathrm{a}, 3 \mathrm{c}$ and $6 \mathrm{~b}$ gives the results with the width $D=1.85,1.5$ and 1.325 , respectively. It means that the greater the scale height, the less the arch expanded. 

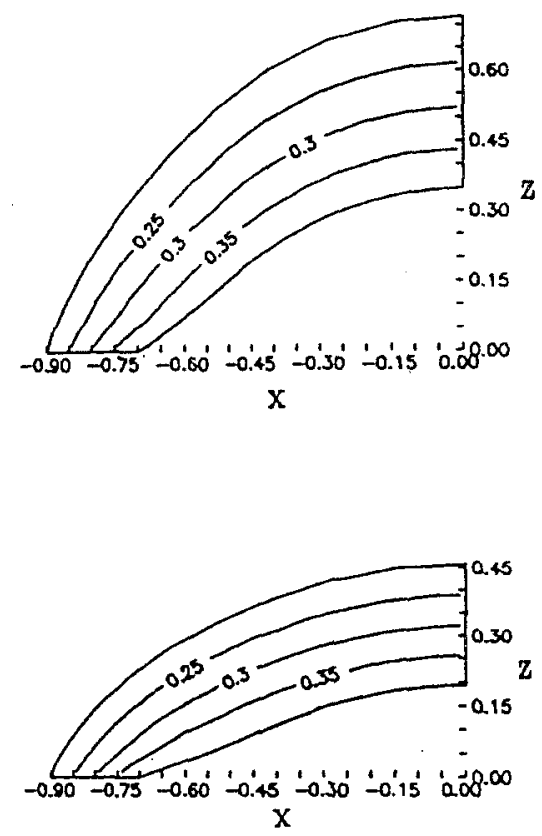

Fig. 6. Magnetic force line with $H=0.55$ (a) and 0.8 (b), respectively, for $\alpha=1$.

\subsection{INFLUENCE OF FORCE-FREE FACTOR $\alpha$}

Owing to the confinement of the atmospheric pressure, the value of $\alpha$ is determined by the photospheric boundary condition, and associated with azimuthal component of field. The case of $\alpha^{2}=2$ is given in Fig. $7 \mathrm{~b}$ for $H=0.65, x_{1}=-0.9$ and $x_{2}=-0.7$ corresponding to the boundary condition in Fig. 7a. Comparing with Fig. $3 c$, the configuration of flux arch in Fig. 7 rises relatively higher.

\section{Discussion and Conclusions}

The finite element method is used succesfully to analyze the equilibrium of confined isolated magnetic arch. The conditions of free boundary surface and the total pressure conservation at free surface made the problem complicated, and the solution is convergent only for certain parameter range. The results show that, numerical simulation in the present paper is effective.

The model in the present study is a discontinuous one. Both the thermodynamical quantities and the magnetic field across the boundary of the arch would be discontinuous and should satisfy the conservation condition of the total pressure. And the photosphere boundary condition we adopted should also consider this condition at the footpoints. This is fully different from the continuous model. The 

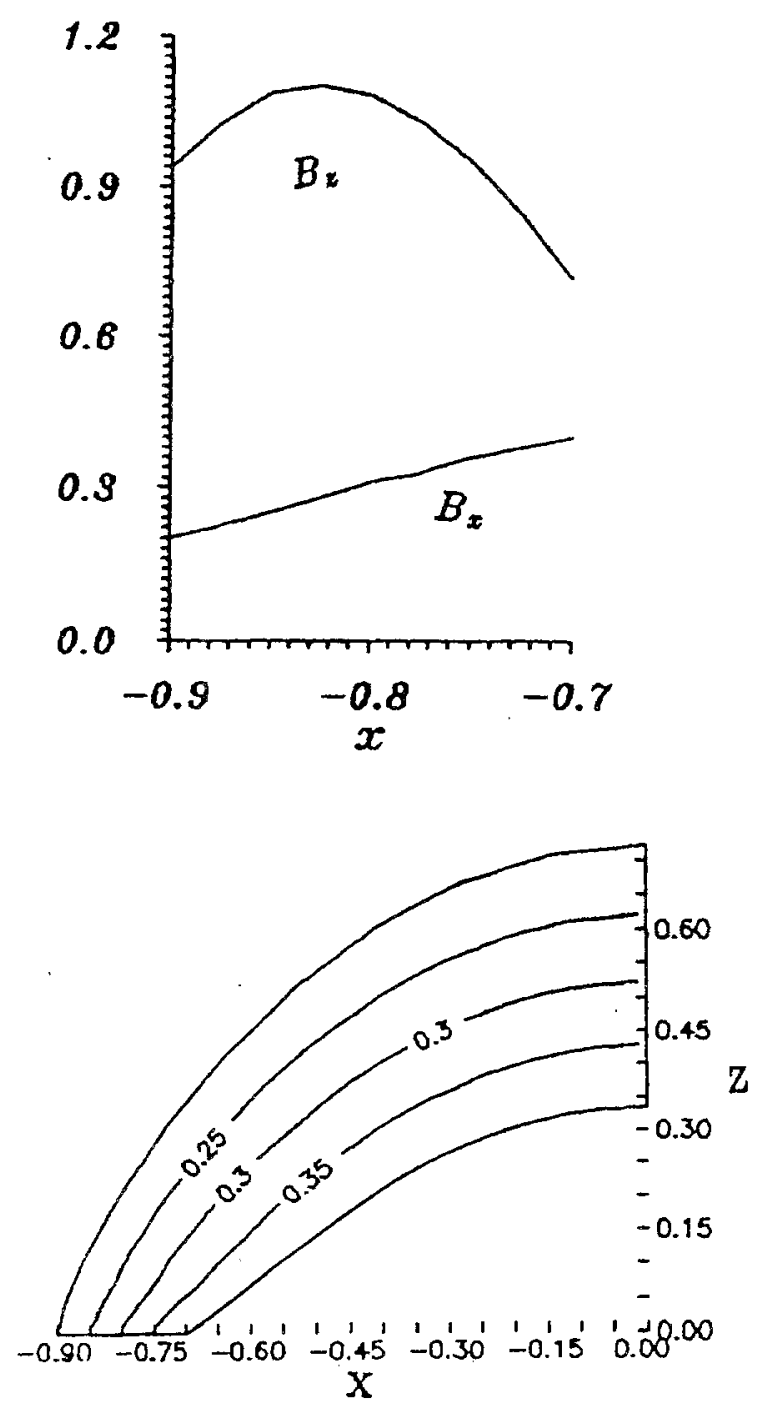

Fig. 7. Magnetic force line (b) corresponding to boundary condition (a) with $h=0.65, \alpha^{2}=2$.

configuration of the magnetic flux arch depends sensitively on the atmosphere scale height. The isolated flux arch appears thinner with the larger scale height, and the greater the photosphere magnetic field (or the ratio of longitudinal magnetic field to transverse field), the higher the tube rises. Besides, the force-free factor and the relative position of the two footpoints also have influence on the shape of arch.

As for the case of the non-isothermal atmosphere, the problem could be considered in a similar approach. Realistic arch in the solar atmosphere is 3-dimensional 
and may not be axi-symmetric. These problems will be studied further and discussed elsewhere.

\section{References}

Spruit, H.: 1981, in: The Sun as a Star, NASA SP-450, p.385.

Parker, E. N.: 1979, Cosmical Magnetic Fields, Clarendon Press.

Hu, W. R. et al.: 1983, Solar Physics 83, 195.

Hu, W. R.: 1987, J. Plasma Physics 37, 257.

Hu, W. R.: 1989, Scientia Sinica 32, 1353.

Deinzer, W. G.: 1965, Astrophys. J. 141, 548.

Osherovich, V. A.: 1974, Solar Physics 94, 205.

Hu, W. R., Li, J. G.: 1987, Scientia Sinica 30, 269.

Zhang, X. and Hu, W. R.: 1992a, Astrophys. Space Sci. 192, 247.

Zhang, X. and Hu, W. R.: 1992b, Scientia Sinica (submitted). 